\title{
Burden of Implanted-Device-Detected Atrial High-Rate Episode Is Associated With Future Heart Failure Events - Clinical Significance of Asymptomatic Atrial Fibrillation in Patients With Implantable Cardiac Electronic Devices -
}

\author{
Ryo Nishinarita, MD; Shinichi Niwano, MD, PhD; Hidehira Fukaya, MD, PhD; \\ Jun Oikawa, MD, PhD; Takeru Nabeta, MD, PhD; Gen Matsuura, MD; \\ Yuki Arakawa, MD; Shuhei Kobayashi, MD; Yuki Shirakawa, MD; \\ Ai Horiguchi, MD; Hironori Nakamura, MD, PhD; Naruya Ishizue, MD, PhD; \\ Jun Kishihara, MD, PhD; Akira Satoh, MD, PhD; Junya Ako, MD, PhD
}

\begin{abstract}
Background: The relationship between atrial high-rate episode (AHRE) burden (i.e., the frequency of atrial tachyarrhythmia) and heart failure (HF) risk is unclear. We hypothesized that new-onset and higher burden of AHRE are associated with HF.
\end{abstract}

\begin{abstract}
Methods and Results: We included 104 consecutive patients with cardiac implantable electronic devices (CIEDs) capable of continuous atrial rhythm monitoring. Patients with AF history were excluded. To stratify patients, AHREs were evaluated only during the initial 1 year after CIED implantation. The primary endpoint was all-cause death or new-onset or worsening HF that required unplanned hospitalization or readjustment of HF drug therapy. At 1 year after CIED implantation, 34/104 patients (33\%) exhibited AHREs. No difference in basal clinical characteristics except for left ventricular ejection fraction between patients with and without new-onset AHREs was found. AHRE groups had more HF events than the non-AHRE group. All patients were divided into 3 groups based on AHRE burden: none, low, and high. Worsening HF was observed in 12 patients (12\%). Cox hazard analysis revealed that AHRE and higher AHRE burden were independent predictive factors for worsening HF. The high group showed a higher risk for HF than the non-AHRE groups, but no significant difference was found between the low- and non-AHRE groups.
\end{abstract}

Conclusions: New-onset higher AHRE burden was associated with subsequent risk for HF in patients with CIEDs.

Key Words: Asymptomatic atrial fibrillation; Atrial fibrillation; Atrial high-rate episodes; Cardiac implantable electronic devices; Heart failure

A trial fibrillation (AF) is a common cardiac tachyarrhythmia that is associated with an increased risk of stroke, heart failure (HF), and death. The presence of AF is reportedly associated with an increased risk of $\mathrm{HF}$, regardless of its clinical pattern.1,2 Changes in clinical AF (i.e., progression to permanent/persistent AF from paroxysmal AF, prolongation of AF duration, and/or increase in the left atrial size, etc.) are clinical predictors of HF. ${ }^{1-5}$ However, clinically detecting AF in the early phase is not always easy, particularly in cases of asymptomatic AF. Although repeat ECG recording or 24-h ambulatory ECG monitoring are considered useful in detecting AF in the early phase, their sensitivities as detection tools are totally limited. ${ }^{4}$

Continuous rhythm monitoring using cardiac implantable electronic devices (CIEDs) has recently been attempted for the diagnosis of brief episodes of arrhythmia, including paroxysmal AF, which are recorded as atrial high-rate episodes (AHREs), particularly in patients without ECGdocumented AF. ${ }^{6}$ Using this technique, new-onset atrial tachyarrhythmia (AT)/AF can be diagnosed earlier in patients with CIEDs than in patients without CIEDs.

AHREs detected using CIEDs are reportedly associated with an increased risk of stroke and systemic embolism, even in patients without any evidence of AF. ${ }^{7}$ Furthermore, AHREs lasting longer ( $>5.5 \mathrm{~h}$ during a 30 -day period) are associated with a double risk of embolic events..$^{89}$ Although Healey et al reported no association between subclinical AF and hospitalization for $\mathrm{HF},{ }^{9}$ more recent studies have indicated that AHREs detected using CIEDs are associated with an increased risk of worsening HF. ${ }^{10,11}$ Therefore, the correlation between AHREs detected using CIEDs and

Received October 25, 2018; revised manuscript received December 25, 2018; accepted January 9, 2019; J-STAGE Advance Publication released online February 26, 2019 Time for primary review: 23 days

Department of Cardiovascular Medicine, Kitasato University School of Medicine, Sagamihara, Japan

Mailing address: Ryo Nishinarita, MD, Department of Cardiovascular Medicine, Kitasato University School of Medicine, 1-15-1 Kitasato, Minami-ku, Sagamihara 252-0374, Japan. E-mail: nishinari.0924@gmail.com

ISSN-1346-9843 All rights are reserved to the Japanese Circulation Society. For permissions, please e-mail: cj@j-circ.or.jp 


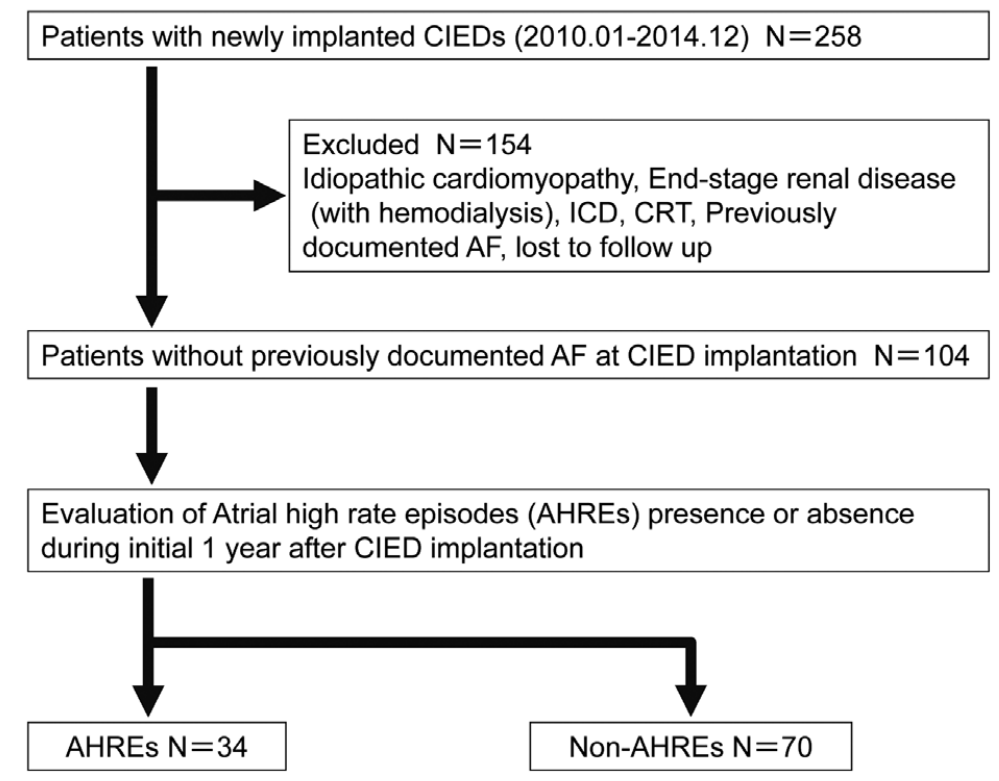

Figure 1. Schematic of patient selection and grouping. AF, atrial fibrillation; CIED, cardiac implantable electronic device; CRT, cardiac resynchronization therapy device; ICD, implantable cardioverter-defibrillator.

patient prognoses still remains controversial. Here, we retrospectively investigated whether new-onset AHREs and their burden within the first year after CIED implantation were associated with a higher risk of worsening $\mathrm{HF}$ in future follow-up of patients with CIEDs.

\section{Methods}

\section{Study Population}

The study population comprised 258 consecutive patients who underwent implantation of new CIEDs between January 2010 and December 2014 at the Kitasato University Hospital. For the purpose of monitoring AHREs, patients without an atrial lead were excluded. The CIEDs used in the present study consisted of a permanent DDD pacemaker for sick sinus syndrome or atrioventricular block. Patients with implantable cardioverter-defibrillators and cardiac resynchronization therapy devices were also excluded because they were expected to have a higher risk for $\mathrm{HF}$ because of their basic cardiac diseases. Each device was implanted in accordance with the class I-IIa indications of the current AHA/JCS guidelines. ${ }^{12}$ Patients with idiopathic cardiomyopathy, endstage renal disease (with hemodialysis), and/or previously documented AF were also excluded. Finally, a total of 104 patients were enrolled in the study (Figure 1).

\section{Data Collection and AF Detection}

In all patients, confirmation of baseline clinical history, 12-lead ECG, chest X-ray, transthoracic echocardiography, and routine blood sampling were performed as the basic examinations prior to CIED implantation. Preceding AF history was confirmed by AF recordings on surface ECG and/or Holter ECG, and such patients were excluded from observation. All CIEDs used were capable of recording AHREs. The CIEDs used were manufactured by St. Jude Medical (Saint Paul, MN, USA) for 32, Medtronic (Minneapolis, MN, USA) for 27, Biotronik (Berlin, Germany) for 19, Ela Medical (Montrouge, France) for 15, and Boston Scientific (Marlborough, MA, USA) for 11 patients. The patients were assessed at 6-month intervals after the initial device check performed 1 week post implantation. Clinical data, including interrogation data and the presence and burden of AHREs during the preceding 6-month period, were collected at the time of device check. AHRE was defined as any episode of sustained AT ( $>5 \mathrm{~min}$ and $>170$ beats $/ \mathrm{min}$ ) detected through the atrial lead. The atrial sensing threshold was set at $0.5 \mathrm{mV}$. To detect AHRE, a cutoff $>5 \mathrm{~min}$ was used in this study for exclusion of over-diagnosis of various atrial signals, such as far-field oversensing and/or sinus tachycardia. ${ }^{13}$ The other atrial noises were excluded by checking intracardiac ECGs stored in the pacemakers as much as possible. ${ }^{10} \mathrm{We}$ divided our study population into patients with and without newly detected AHREs during the first year after CIED implantation. All patients were subsequently observed for $65 \pm 18$ months.

\section{Definition of Clinical Endpoints}

The primary endpoint was defined as all-cause death or new-onset or worsening $\mathrm{HF}$, all of which required unplanned hospitalization or readjustment of drug therapy for HF. Fatal arrhythmia and/or cerebral infarction was additionally considered as a secondary endpoint. HF was diagnosed based on the major and minor clinical criteria described by the Framingham Heart Study. ${ }^{\mathbf{1 4}}$ These endpoints were evaluated in all patients from the subsequent observation period to the initial 1-year observation after CIED implantation.

\section{Grouping of Patients}

The appearance and/or burden of AHREs was evaluated in the first year after CIED implantation and all patients were grouped as with or without AHREs. The baseline clinical characteristics and the incidences of the endpoints were compared between groups. In addition, to observe the influence of AHRE burden on worsening HF, patients with AHREs were further divided into 2 groups based on 


\begin{tabular}{|c|c|c|c|c|}
\hline & $\begin{array}{c}\text { Total } \\
(n=104)\end{array}$ & $\begin{array}{c}\text { AHREs } \\
(n=34)\end{array}$ & $\begin{array}{l}\text { None } \\
(n=70)\end{array}$ & $P$ value \\
\hline Age (years) & $75.1 \pm 10.9$ & $76.7 \pm 10.0$ & $74.3 \pm 11.3$ & 0.37 \\
\hline Sex, males, n (\%) & $53(51.0)$ & $16(43.2)$ & $37(55.2)$ & 0.24 \\
\hline \multicolumn{5}{|l|}{ Diagnosis } \\
\hline SSS, n (\%) & $41(39.4)$ & $18(48.7)$ & $23(34.3)$ & \multirow{2}{*}{0.15} \\
\hline AVB, n (\%) & $63(60.6)$ & $19(51.4)$ & $44(65.7)$ & \\
\hline \multicolumn{5}{|l|}{ History } \\
\hline Prior cerebral infarction, n (\%) & $4(3.9)$ & $2(5.4)$ & $2(3.0)$ & 0.54 \\
\hline Prior CHF, n (\%) & $18(17.3)$ & $7(18.9)$ & $11(16.4)$ & 0.75 \\
\hline Prior IHD, n (\%) & $15(14.4)$ & $7(18.9)$ & $8(11.9)$ & 0.33 \\
\hline \multicolumn{5}{|l|}{ Medications } \\
\hline$\beta$-blocker, n (\%) & $12(11.5)$ & $3(8.1)$ & $9(13.4)$ & 0.42 \\
\hline RASI, n (\%) & $57(54.8)$ & $23(62.3)$ & $34(50.8)$ & 0.26 \\
\hline Antiplatelet drugs, n (\%) & $20(19.2)$ & $5(13.5)$ & $15(22.4)$ & 0.27 \\
\hline Anticoagulant drugs, n (\%) & $11(10.6)$ & $5(13.5)$ & $6(9.0)$ & 0.47 \\
\hline \multicolumn{5}{|l|}{ Percentage of atrial pacing } \\
\hline$<40, \mathrm{n}(\%)$ & $53(51.0)$ & $17(46.0)$ & $36(53.7)$ & \multirow{3}{*}{0.1} \\
\hline 40-70, n (\%) & $27(26.0)$ & $14(37.8)$ & $13(19.4)$ & \\
\hline$>70, \mathrm{n}(\%)$ & $24(23.1)$ & $6(16.2)$ & $18(26.9)$ & \\
\hline \multicolumn{5}{|l|}{ Percentage of ventricular pacing } \\
\hline$<40, \mathrm{n}(\%)$ & $34(32.7)$ & $13(35.1)$ & $21(31.3)$ & \multirow{3}{*}{0.92} \\
\hline $40-70$, n (\%) & $3(2.9)$ & $1(2.7)$ & $2(3.0)$ & \\
\hline$>70 \%, \mathrm{n}(\%)$ & $67(64.4)$ & $23(62.2)$ & $44(65.7)$ & \\
\hline \multicolumn{5}{|l|}{ Laboratory data } \\
\hline $\mathrm{Cr}(\mathrm{mg} / \mathrm{dL})$ & $0.98 \pm 0.47$ & $1.11 \pm 0.67$ & $0.92 \pm 0.32$ & 0.47 \\
\hline LDL-C (mg/dL) & $110 \pm 34.4$ & $106 \pm 31.1$ & $112 \pm 35.8$ & 0.53 \\
\hline \multicolumn{5}{|l|}{ Echocardiography } \\
\hline LAD (mm) & $38.7 \pm 6.1$ & $39.3 \pm 6.0$ & $38.4 \pm 6.2$ & 0.41 \\
\hline LVEF (\%) & $65.6 \pm 7.2$ & $68.5 \pm 7.7$ & $64.3 \pm 6.7$ & $0.006^{*}$ \\
\hline MR, n (\%) & $5(4.8)$ & $1(2.9)$ & $4(5.7)$ & 0.54 \\
\hline TR, n (\%) & $7(6.7)$ & $3(8.8)$ & $4(5.7)$ & 0.55 \\
\hline
\end{tabular}

*Statistical significance. AHRE, atrial high-rate episode; AVB, atrioventricular block; CHF, congestive heart failure; IHD, ischemic heart disease; LAD, left atrial dimension; LDL-C, low-density lipoprotein cholesterol; LVEF, left ventricular ejection fraction; MR, mitral regurgitation; RASI, renin-angiotensin system inhibitor; SSS, sick sinus syndrome; TR, tricuspid regurgitation.

\begin{tabular}{|lcccc|}
\hline \multicolumn{4}{|l|}{ Table 2. Cumulative Clinical Outcome at $\mathbf{7}$ Years for Patients With and Without AHREs } & \\
& $\begin{array}{c}\text { All } \\
(\mathbf{n = 1 0 4 )}\end{array}$ & $\begin{array}{c}\text { AHRE } \\
(\mathbf{n = 3 4 )}\end{array}$ & $\begin{array}{c}\text { Non-AHRE } \\
(\mathbf{n = 7 0 )}\end{array}$ & P value \\
Worsening HF, $\mathrm{n}(\%)$ & $12(11.5)$ & $8(23.5)$ & $4(5.7)$ & $0.008^{*}$ \\
All-cause death, $\mathrm{n}(\%)$ & $9(8.7)$ & $6(17.7)$ & $3(4.4)$ & $0.02^{*}$ \\
Fatal arrhythmia, $\mathrm{n}(\%)$ & $2(1.9)$ & $2(5.9)$ & $0(0)$ & $0.04^{*}$ \\
Cerebral infarction, $\mathrm{n}(\%)$ & $2(1.9)$ & $2(5.9)$ & $0(0)$ & $0.04^{*}$ \\
Clinical AF development, $\mathrm{n}(\%)$ & $12(11.5)$ & $8(23.5)$ & $4(5.7)$ & $0.008^{*}$ \\
Bleeding event, $\mathrm{n}(\%)$ & $11(10.6)$ & $6(17.7)$ & $5(7.1)$ & 0.1 \\
\hline
\end{tabular}

$\mathrm{AF}$, atrial fibrillation; AHRE, atrial high-rate episode; $\mathrm{HF}$, heart failure.

the AHRE burden using the median value of the recorded AHREs. The study population was divided into 3 groups based on AHRE burden, namely, the non-AHRE group (no AHREs detected), low-AHRE group (AHRE burden $\leq$ median AHREs), and high-AHRE group (AHRE burden $>$ median AHREs). Finally, the incidence of worsening HF was compared among the 3 AHRE groups to evaluate the effect of the AHRE burden.

\section{Statistical Analysis}

Data were analyzed using JMP software (SAS Institute, Cary, NC, USA). Continuous variables were compared using an independent t-test or the Mann-Whitney U-test. Categorical data were analyzed using the chi-square test. Cumulative hazard curves were modeled using the KaplanMeier method and compared using a log-rank test. Cox proportional hazards modeling was used to adjust for baseline imbalances with respect to worsening HF, transient 
(A) HF worsening events

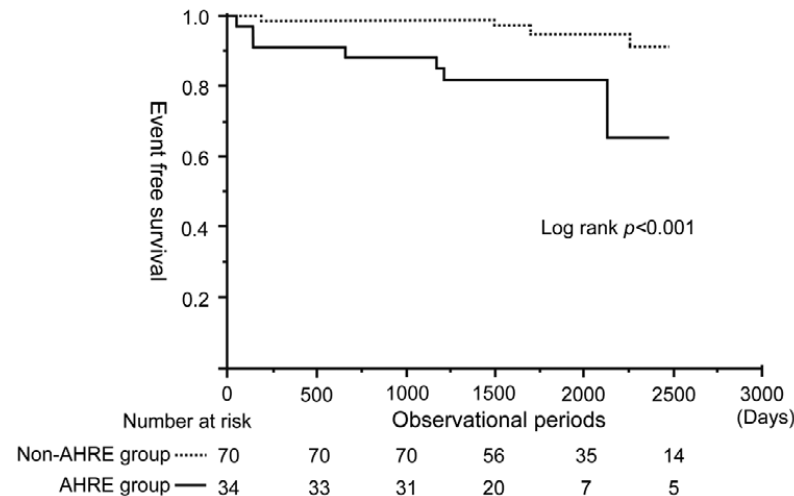

(B) All cause death

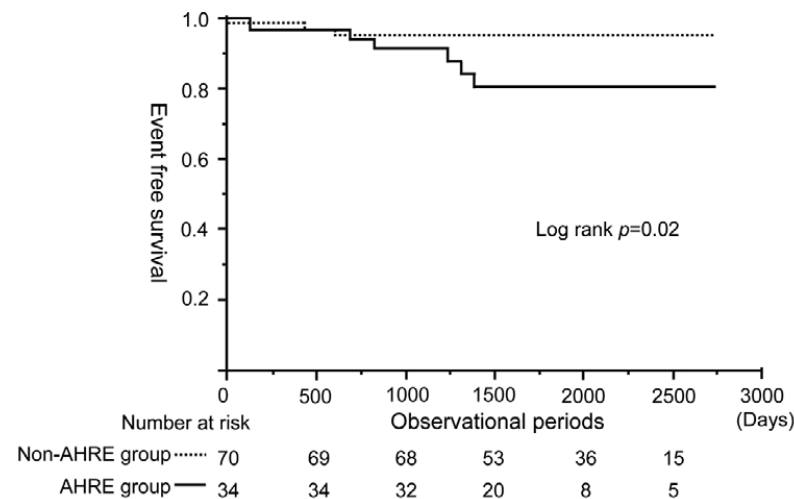

(C) Fatal arrhythmia

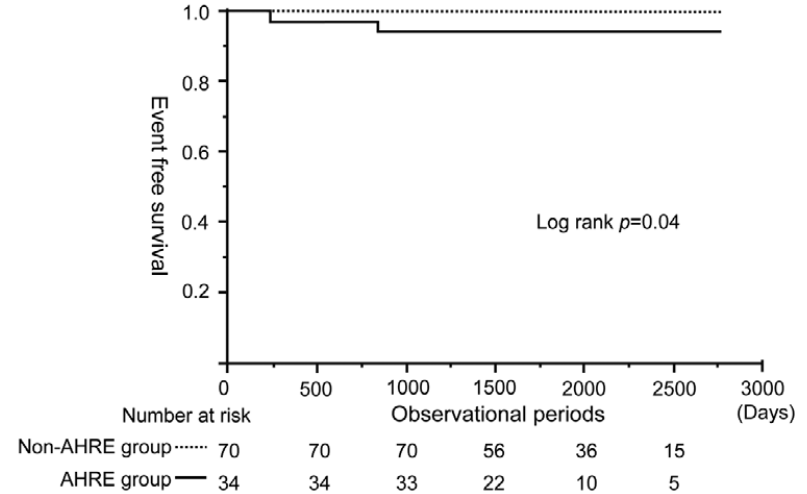

(D) Cerebral infraction

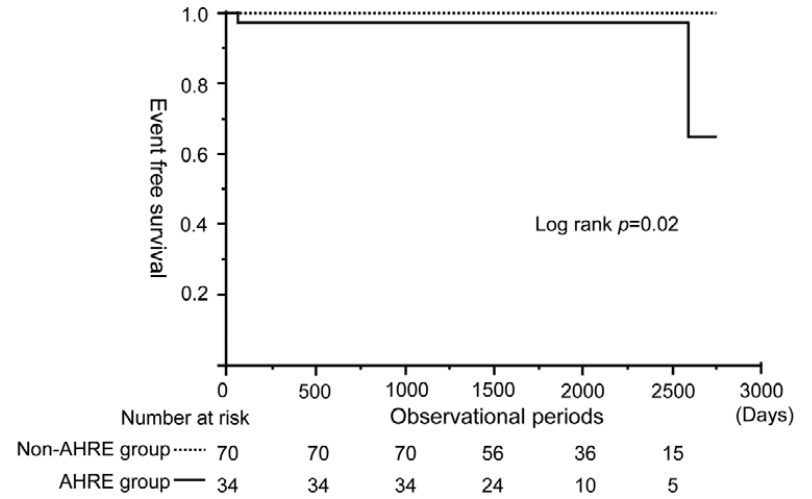

Figure 2. Kaplan-Meier analysis for $(\mathbf{A})$ worsening heart failure, $(\mathbf{B})$ all-cause death, $(\mathbf{C})$ fatal arrhythmia and (D) cerebral infarction according to AHREs based on continuous monitoring by CIED. AHRE, atrial high-rate episode; CIED, cardiac implantable electronic device.

ischemic attacks, sinus node dysfunction, atrioventricular block, hypertension, diabetes mellitus, dyslipidemia, ischemic heart disease history, age, sex, atrial or ventricular pacing rate, laboratory findings and echocardiography parameters, and usage of $\beta$-blockers, angiotensin-converting enzyme inhibitors or angiotensin II receptor blockers, and antithrombotic therapy. For all comparisons, $\mathrm{P}<0.05$ was considered statistically significant.

\section{Results}

\section{Incidence and Burden of AHREs}

Of the 104 patients, 34 exhibited AHREs during the first year after CIED implantation; these 34 patients were subsequently allocated to the AHRE group, and the remaining 70 patients were allocated to the non-AHRE group. In the AHRE group, the AHRE burden was $70 \pm 140 \mathrm{~h}$ (range, 2.2-612; median, $22 \mathrm{~h}$ ) during the 6-month period. Using the median AHRE (i.e., $22 \mathrm{~h} / 6$ months), the AHRE group was further divided into 2 subgroups. Finally, the study population was divided into 3 groups according to AHRE burden: non-AHRE group, no AHRE episodes $(\mathrm{n}=70,67 \%)$; low-AHRE group, AHRE burden $\leq 22 \mathrm{~h}$ $(\mathrm{n}=14,14 \%)$; and high-AHRE group, AHRE burden $>22 \mathrm{~h}$ $(\mathrm{n}=20,19 \%)$.

\section{Comparison of Basic Characteristics}

Table 1 presents the basic clinical characteristics of the patients before CIED implantation. Statistical differences were analyzed between the AHRE and non-AHRE groups. Although the left ventricular ejection fraction (LVEF) was higher in the AHRE group than in the non-AHRE group, the other parameters did not exhibit any statistical differences.

\section{Clinical Endpoints According to New-Onset AHRE}

As the primary endpoint, all-cause death occurred in 6/34 $(17.7 \%)$ patients in the AHRE group and in $3 / 70(4.4 \%)$ in the non-AHRE group ( $\mathrm{P}=0.02$, Table 2). Worsening HF occurred in $8 / 34(23.5 \%)$ patients in the AHRE group and in $4 / 70(5.7 \%)$ in the non-AHRE group $(\mathrm{P}=0.008$, Table 2$)$. As the secondary endpoint, fatal arrhythmia occurred in $2 / 34(5.9 \%)$ patients in the AHRE group and in $0 / 70(0 \%)$ in the non-AHRE group $(\mathrm{P}=0.04$, Table 2). Cerebral infarction occurred in $2 / 34(5.9 \%)$ patients in the AHRE group and in $0 / 70(0 \%)$ in the non-AHRE group $(\mathrm{P}=0.04$, Table 2). A total of 12/104 (11.5\%) patients exhibited ECG-documented AF within the first year after CIED implantation: $8 / 34(23.5 \%)$ patients in the AHRE group and $4 / 70(5.7 \%)$ in the non-AHRE group ( $\mathrm{P}=0.008$, Table 2$)$. The Kaplan-Meier curves demonstrated that compared 
Table 3. Independent Predictors of HF Following Implantation of Permanent Pacemaker

\begin{tabular}{|c|c|c|c|c|}
\hline \multirow{2}{*}{ Parameter } & \multicolumn{2}{|c|}{ Univariate analysis } & \multicolumn{2}{|c|}{ Multivariate analysis } \\
\hline & HR (95\% Cl) & $P$ value & HR (95\% Cl) & $P$ value \\
\hline Age (years) & $1.00(0.95-1.06)$ & 0.90 & & \\
\hline Sex, males, n (\%) & $0.60(0.18-1.92)$ & 0.39 & & \\
\hline \multicolumn{5}{|l|}{ Diagnosis } \\
\hline SSS, n (\%) & $1.61(0.49-5.1)$ & 0.42 & & \\
\hline AVB, n (\%) & $0.63(0.2-2.0)$ & 0.42 & & \\
\hline \multicolumn{5}{|l|}{ History } \\
\hline Hypertension, n (\%) & $0.85(0.27-3.2)$ & 0.80 & & \\
\hline Prior cerebral infarction, $\mathrm{n}(\%)$ & $2.70(0.14-14.1)$ & 0.42 & & \\
\hline Prior CHF, n (\%) & $7.64(2.42-25.9)$ & $<0.001^{*}$ & $6.7(1.73-30.9)$ & $0.006^{*}$ \\
\hline Prior IHD, n (\%) & $2.13(0.47-7.23)$ & 0.29 & & \\
\hline \multicolumn{5}{|l|}{ Medications } \\
\hline RASI, n (\%) & $1.62(0.51-6.07)$ & 0.42 & & \\
\hline$\beta$-blockers, $\mathrm{n}(\%)$ & $0.74(0.04-3.82)$ & 0.76 & & \\
\hline Antiplatelet drugs, n (\%) & $1.36(0.30-4.57)$ & 0.65 & & \\
\hline Anticoagulant drugs, $\mathrm{n}(\%)$ & $3.98(2.28-12.8)$ & 0.08 & & \\
\hline Percentage of atrial pacing, $\mathrm{n}(\%)$ & $1.00(0.99-1.02)$ & 0.78 & & \\
\hline Percentage of ventricular pacing, $n(\%)$ & $1.00(0.99-1.02)$ & 0.68 & & \\
\hline AHRE (\%) & $1.18(1.04-1.31)$ & $0.02^{*}$ & $1.27(1.11-1.5)$ & $0.004^{*}$ \\
\hline \multicolumn{5}{|l|}{ Laboratory data } \\
\hline $\mathrm{Cr}(\mathrm{mg} / \mathrm{dL})$ & $5.03(2.36-10.5)$ & $<0.001^{\star}$ & $3.82(1.74-8.4)$ & $0.002^{*}$ \\
\hline LDL (mg/dL) & $0.99(0.98-1.01)$ & 0.59 & & \\
\hline \multicolumn{5}{|l|}{ Echocardiography } \\
\hline LAD (mm) & $0.96(0.86-1.05)$ & 0.37 & & \\
\hline LVDd (mm) & $0.97(0.89-1.07)$ & 0.65 & & \\
\hline LVEF (\%) & $0.98(0.91-1.06)$ & 0.63 & & \\
\hline MR, n (\%) & $1.54(0.08-8.00)$ & 0.69 & & \\
\hline TR, n (\%) & $1.02(0.69-5.35)$ & 0.98 & & \\
\hline
\end{tabular}

$\mathrm{Cl}$, confidence interval; HR, hazard ratio; LDL, low-density lipoprotein; LVDd, left ventricular diastolic dimension. Other abbreviations as in Tables 1,2.

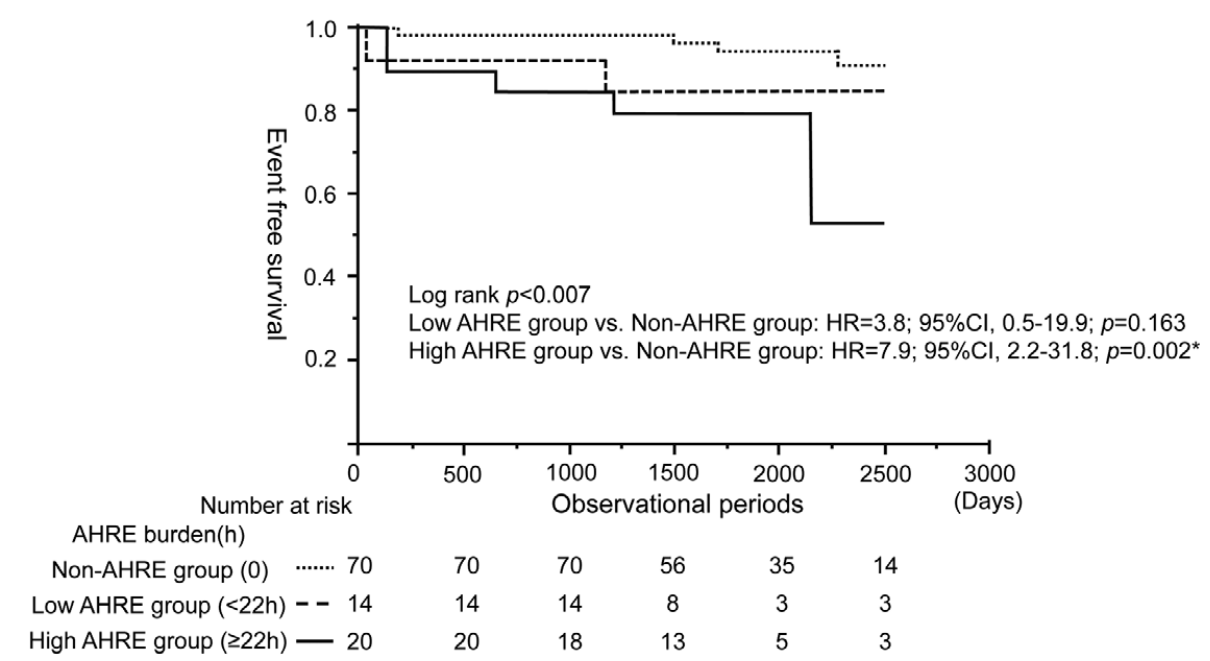

Figure 3. Kaplan-Meier analysis for worsening of heart failure stratified by time-dependent duration according to AHREs based on continuous monitoring by CIED. AHRE, atrial high-rate episode; CIED, cardiac implantable electronic device. 
with the non-AHRE group, the AHRE group experienced a higher rate of worsening $\mathrm{HF}$ (log-rank, $\mathrm{P}<0.001)$, all-cause death $(\mathrm{P}=0.02)$, fatal arrhythmia $(\mathrm{P}=0.04)$, and cerebral infarction ( $\mathrm{P}=0.02)$ (Figure 2).

\section{Association Between AHRE Burden and Clinical Endpoints}

Worsening HF occurred in $12 / 104$ patients $(12 \%)$ during the follow-up period. Prior congestive HF, presence of AHREs, and serum creatinine $(\mathrm{Cr} ; \mathrm{mg} / \mathrm{dL})$ were the significant univariate predictors of worsening HF. The multivariate Cox regression model revealed the following as independent predictors for worsening HF: prior congestive HF (hazard ratio [HR] 6.7, 95\% confidence interval [CI] 1.73-30.9, $\mathrm{P}=0.006$ ), presence of AHREs (HR 1.27, 95\% CI 1.11-1.5, $\mathrm{P}=0.004)$, and serum $\mathrm{Cr}$ (HR 3.82, 95\% CI 1.74-8.4, $\mathrm{P}=0.002$ ). In the multivariate analysis, when comparing the 3 groups (none, low, and high AHRE burden), the strongest independent predictors for worsening HF were the presence of AHREs and AHRE burden (Table 3). The Kaplan-Meier curves indicated that the high-AHRE burden group had a higher risk for worsening HF (HR 7.9, 95\% CI, 2.2-31.8, $\mathrm{P}=0.002$ ) compared with the non-AHRE group. In contrast, there was no significant difference between the low-AHRE and non-AHRE groups (Figure 3).

\section{Discussion}

In the present study we demonstrated that the detection of AHREs during the first year after CIED implantation was associated with the incidence of future cardiovascular events, such as worsening HF, all-cause death, fatal arrhythmia, and cerebral infarction. The presence of AHREs was an independent predictive factor for worsening HF during the subsequent long-term observation period. Additionally, patients with a higher device-detected AHRE burden lasting for $>22 \mathrm{~h}$ during a 6 -month period were 7.9fold more likely to suffer from worsening HF than patients without detected AHREs, although patients with a lower AHRE burden lasting for $\leq 22 \mathrm{~h}$ did not exhibit any increase in their risk for worsening HF.

HF incidence in patients with CIEDs was reportedly observed in 3-12\% of all patients over a mean 3-year follow-up period. ${ }^{15}$ In particular, patients with CIEDs with LV dysfunction ( $\mathrm{EF}<50 \%$ ) and a high rate of ventricular pacing exhibited a dramatic increase in the risk of HF events. In contrast, patients with CIEDs with a low-risk background, such as with preserved LVEF, no history of HF or myocardial infarction, normal baseline QRS duration, or well-tolerated ventricular desynchronization therapy, exhibited a considerably lower risk of new-onset HF. ${ }^{16}$ In the present study, the incidence of HF in patients with CIEDs was $11.5 \%$, within the reported range, although we included patients with a relatively low risk for HF (i.e., with preserved $\mathrm{EF}$ and/or a low percentage of ventricular pacing $(25.7 \%$ patients had ventricular pacing $>70 \%)$ ).

A recent meta-analysis demonstrated significant increases in the risk of worsening $\mathrm{HF}$ with $\mathrm{AF}$ in asymptomatic (stabilized) HF patients. ${ }^{17}$ Other prospective studies reported that $\mathrm{AF}$ is associated with an increase in the long-term risk for HF. ${ }^{18,19}$ These studies emphasized the importance of earlier AF detection for predicting clinical HF. The utility of conventional methods, including ECG and Holter ECG monitoring, is totally limited for early detection of AF because of the restricted time available for monitoring. In this study, we could detect a considerable number of patients with new-onset AHREs in the relatively early phase after CIED implantation, and such devicedetected AHREs were associated with future worsening of HF. We used the criterion of AHREs as atrial rate $>170$ beats/min lasting $>5 \mathrm{~min}$ and the median value of an AHRE burden of $22 \mathrm{~h}$ was used as the borderline between the high- and low-AHRE burden groups. In preceding reports, AHRE was defined as episodes lasting $>5-6 \mathrm{~min}$ with an atrial rate $>170-190$ beats/min, ${ }^{6,8,14}$ which similar to our definition. By using this criterion, the other researchers reported that overdetection of AHREs because of various atrial noise signals could be excluded as much as possible. The detection rate of AHREs was $33 \%$ in the present study, which was within the reported range of such detections over a 2-year observation period (i.e., 20-60\%). ${ }^{20-22}$ Although the presence of AHREs does not directly indicate the presence of clinical or ECG-documented AF, our data suggested the possibility of early detected new-onset AHREs in CIED patients as a predictor for future HF and/or worsening HF events. Moreover, we successfully documented different contributions of higher and lower burdens of device-detected AHREs in patients with CIEDs. We found that patients with a higher AHRE burden exhibited more HF worsening events than did patients without AHREs; however, the patients with a lower AHRE burden did not show any such tendency, which might have resulted from the small patient population. This indicates that compared with evaluating the presence or absence of AHREs, evaluating the AHRE burden may more precisely predict future HF events. Three possible explanations can be given for the mechanism of the correlation between device-detected AHREs and HF worsening. First, AF itself may induce LV dysfunction. Although the detected AHREs may not correlate with the presence of AF, the presence of detected AHREs strongly relates to the presence of clinical AF. ${ }^{16}$ Irregularity of atrial contraction loses the atrial contribution to the cardiac output and rapid irregular ventricular rhythm increases the load on the ventricle. Such a disadvantage may result in LV dysfunction depending on the AF and/or AHRE burden.,10 Second, AF induces atrial remodeling, which may then disturb atrial electrical and mechanical function. ${ }^{9,23}$ Even if AF and/or AHREs are asymptomatic, structural changes, such as atrial enlargement and atrial wall fibrosis, can be induced and may result in atrial contraction disturbance as well as mitral regurgitation development. These changes will also increase the load on the ventricle. ${ }^{1}$ Third, AHREs and/or AF may appear as surrogate markers of the progression of LV dysfunction caused by any other pathological conditions. In this case, AHREs are considered to appear under the influence of LV dysfunction; thus, the relationship between an increase in the AHRE burden and the progression of LV dysfunction should be well explained. ${ }^{24}$ In this study, we did not have data (transthoracic echocardiography, and blood sampling etc.) after mid- and long-term follow-up observations and thus cannot discuss the relationship between temporal AHREs and cardiac function assessment. However, the mechanism may possibly only apply in the early stage after CIEDs implantation. We could possibly predict the risk of future worsening HF by estimating the AHRE burden in the early stage after CIED implantation. Consequently, asymptomatic AF with a long-lasting burden should be considered for early intervention, including medication and catheter ablation, because of the risk of 
worsening $\mathrm{HF}$.

\section{Study Limitations}

There were several limitations to mention. First, this was non-randomized, retrospective, and observational study with a small number of patients, which may have significantly affected the results owing to confounding factors. In particular, the analysis of the optimal boundary value of AHRE burden may need to be considered in a larger number of patients. In addition, a relatively small number of variables were included in the multivariate Cox proportional hazard model, which may have reduced the detection power and possibly influenced the validity of interactions. Second, given that this was a single-center study and performed in a Japanese population, the number of analyzed patients was relatively small. For more precise validation, a prospective study with a larger number of patients is required. Third, atrial undersensing can occur during AHREs, which can lead to either a failure to detect an AHRE or truncation of a single AHRE into multiple shorter episodes. Moreover, we could not analyze the duration of AHREs in detail as a device parameter in this study because its extraction was difficult. Finally, there was the slightly configuration difference in AHRE detection because of the different pacemaker manufacturers. For more precise validation, a prospective study with a larger number of patients is required. ${ }^{19}$

\section{Conclusions}

In patients with a CIED without clinical AF, new-onset AHREs identified as asymptomatic AF were detected in $32.7 \%$ of the patients during the first year after CIED implantation. Furthermore, the higher AHRE burden was more strongly associated with future risk for HF worsening in patients with a CIED.

\section{Funding / Grants}

None.

\section{Acknowledgments}

We sincerely thank the members of the Department of Cardiovascular Medicine, Kitasato University School of Medicine.

\section{References}

1. Taillandier S, Bernard AB, Lallemand B, Simeon E, Pericart L, Clementy N, et al. Prognosis in patients hospitalized with permanent and nonpermanent atrial fibrillation in heart failure. $\mathrm{Am} \mathrm{J}$ Cardiol 2014; 113: 1189-1195.

2. Koitabashi T, Inomata T, Niwano S. Decompensation is a predictor of poor prognosis in chronic heart failure. Circ J 2005; 69: $823-830$

3. Linssen GCM, Rienstra M, Jaarsma T, Voors A, Van Gelder I, Hillege $\mathrm{H}$, et al. Clinical and prognostic effects of atrial fibrillation in heart failure patients with reduced and preserved left ventricular ejection fraction. Eur J Heart Fail 2011; 13: 1111-1120.

4. Pillarisetti J, Patel A, Boc K, Bommana S, Sawers Y, Vanga S, et al. Evolution of paroxysmal atrial fibrillation to persistent or permanent atrial fibrillation: Predictors of progression. J Atr Fibrillation 2009; 1: 388-394.

5. B Schnabel R, Pecen L, Engler D, Lucerna M, Sellal J, Ojeda F, et al. Atrial fibrillation patterns are associated with arrhythmia progression and clinical outcomes. Heart 2018; 104: 1608-1614.

6. Israel CW, Grönefeld G, Ehrlich JR, Li YG, Hohnloser SH. Long-term risk of recurrent atrial fibrillation as documented by an implantable monitoring device: Implications for optimal patient care. J Am Coll Cardiol 2004; 43: 47-52.

7. Van Gelder IC, Healey JS, Crijns H, Wang J, Hohnloser S, Gold $\mathrm{M}$, et al. Duration of device-detected subclinical atrial fibrillation and occurrence of stroke in ASSERT. Eur Heart J 2017; 38: $1339-1344$.

8. Glotzer TV, Daoud EG, Wyse DG, Singer DE, Ezekowitz MD, Hilker C, et al. The relationship between daily atrial tachyarrhythmia burden from implantable device diagnostics and stroke risk: The TRENDS study. Circ Arrhythmia Electorophysiol 2009; 2: $474-481$.

9. Healey J, Connolly S, Gold M, Israel C, Gelder I, Capucci A, et al. Subclinical atrial fibrillation and the risk of stroke. $N$ Engl $J$ Med 2012; 366: 120-129.

10. Ogino $\mathrm{Y}$, Ishikawa $\mathrm{T}$, Ishigami $\mathrm{T}$, Matsumoto $\mathrm{K}$, Hosoda J, Iguchi $\mathrm{K}$, et al. Characteristics and prognosis of pacemakeridentified new-onset atrial fibrillation in Japanese people. Circ $J$ 2017; 81: 794-798.

11. Kim BS, Chun KJ, Park S, Park K, Kim JS, On YK. Predictors and long-term clinical outcomes of newly developed atrial fibrillation in patients with cardiac implantable electronic devices. Medicine (Baltimore) 2016; 95: e4181.

12. Tracy CM, Epstein AE, Darbar D, Dimarco J, Dunbar S, Estes N, et al. 2012 ACCF/AHA/HRS focused update incorporated into the ACCF/AHA/HRS 2008 guidelines for device-based therapy of cardiac rhythm abnormalities: A report of the American college of cardiology foundation/American heart association task force on practice guide. J Am Coll Cardiol 2013; 61: e6-e75.

13. Pollack WM, Simmons JD, Interian A, Interian A Jr, Atapattu SA, Castellanos A, et al. Clinical utility of intraatrial pacemaker stored electrograms to diagnose atrial fibrillation and flutter. Pacing Clin Electrophysiol 2001; 24: 424-429.

14. Wang TJ, Larson MG, Levy D, Vasan RS, Leip EP, Wolf PA, et al. Temporal relations of atrial fibrillation and congestive heart failure and their joint influence on mortality: The Framingham Heart Study. Circulation 2003; 107: 2920-2925.

15. Mazza A, Bendini MG, Leggio M, Riva U, Ciardiello C, Valsecchi $\mathrm{S}$, et al. Incidence and predictors of heart failure hospitalization and death in permanent pacemaker patients: A single-centre experience over medium-term follow-up. Europace 2013; 15: 1267-1272.

16. Glotzer T, Hellkamp A, Zimmerman J, Sweeney M, Yee R, Marinchak R, et al. Atrial high rate episodes detected by pacemaker diagnostics predict death and stroke: Report of the atrial diagnostics ancillary study of the MOde Selection Trial (MOST). Circulation 2003; 107: 1614-1619.

17. Yang H, Negishi K, Otahal P, Marwick TH. Clinical prediction of incident heart failure risk: A systematic review and metaanalysis. Open Heart 2015; 2: e000222.

18. Stewart S, Hart CL, Hole DJ, McMurray JJ. A population-based study of the long-term risks associated with atrial fibrillation: 20-Year Follow-up of the Renfrew/Paisley study. Am J Med 2002; 113: 359-364.

19. Ohsawa M, Okamura T, Tanno K, Ogasawara K. Risk of stroke and heart failure attributable to atrial fi brillation in middle-aged and elderly people: Results from a five-year prospective cohort study of Japanese community dwellers. J Epidemiol 2017; 27: $360-367$.

20. Cheung JW, Keating RJ, Stein KM, Markowitz S, Iwai S, Shah $\mathrm{B}$, et al. Newly detected atrial fibrillation following dual chamber pacemaker implantation. J Cardiovasc Electrophysiol 2006; 17: $1323-1328$

21. Orlov MV, Ghali JK, Araghi-Niknam M, Sherfesee L, Sahr D, Hettrick DA. Asymptomatic atrial fibrillation in pacemaker recipients: Incidence, progression, and determinants based on the atrial high rate trial. Pacing Clin Electrophysiol 2007; 30: 404-411.

22. Radeljić V, Pavlović N, Manola Š, Delić-Brkljačić D, Pintarić H, Petrač D. Incidence and predictors of asymptomatic atrial fibrillation in patients older than 70 years with complete atrioventricular block and dual chamber pacemaker implantation. Croat Med J 2011; 52: 61 -67.

23. Dilaveris PE, Kennedy HL. Silent atrial fibrillation: Epidemiology, diagnosis, and clinical impact. Clin Cardiol 2017; 40: 413-418.

24. Everett TH 4th, Olgin JE. Atrial fibrosis and the mechanisms of atrial fibrillation. Heart Rhythm 2007; 4(Suppl): S24-S27. 